(C) 2016 IEEE. Personal use of this material is permitted. Permission from IEEE must be obtained for all other uses, in any current or future media, including reprinting/republishing this material for advertising or promotional purposes, creating new collective works, for resale or redistribution to servers or lists, or reuse of any copyrighted component of this work in other works. 
TITLE

A filter-integrated method to improve the radiation performance of planar UWB antenna

AUTHORS

M. -C. Tang

${ }^{1}$ No. 24 Research Institute, China Electronics Technology Group Corporation, Chongqing, 400060, China

${ }^{2}$ College of Communication Engineering, Chongqing University, Chongqing, 400044, China tangmingchun@cqu.edu.cn

$\underline{\text { T. Shi }}$

College of Communication Engineering, Chongqing University, Chongqing, 400044, China 1614824343@qq.com

R. W. Ziolkowski

${ }^{1}$ The Department of Electrical and Computer Engineering, University of Arizona, Tucson, AZ 85721 USA

${ }^{2}$ The Global Big Data Technologies Centre, University of Technology Sydney (UTS), Sydney, Australia

ziolkowski@ece.arizona.edu

M. -C. Tang IS THE CORRESPONDING AUTHOR AND THE PRESENTING AUTHOR

Tel +862365102235

tangmingchun@cqu.edu.cn 


\title{
A filter-integrated method to improve the radiation performance of planar UWB antenna
}

\author{
Ming-Chun Tang ${ }^{1,2}$, Ting $\mathrm{Shi}^{2}$, and Richard W. Ziolkowski ${ }^{3,4}$ \\ 1. No. 24 Research Institute, China Electronics Technology Group Corporation, Chongqing, 400060, \\ China \\ 2. The College of communication engineering, Chongqing university, 400044, Chongqing, China \\ 3. The Department of Electrical and Computer Engineering, University of Arizona, Tucson, AZ, 85721 \\ USA \\ 4. The Global Big Data Technologies Centre, University of Technology Sydney (UTS), Sydney, \\ Australia
}

In this talk, we will propose a filter-integrated method to improve the stability of the radiation patterns of the compact planar UWB antenna, especially increasing the broadside realized gain values at the higher portion of the UWB frequency range. As is known, with the rapid development of UWB technology for commercial communication applications, many practical applications, such as portable devices, high-accuracy positioning systems, cognitive radios and so on, require constant realized gains in a specified direction. However, the radiation pattern degradation in the upper portion of the UWB frequency range is a serious defect of the planar designs.

In order to alleviate this defect, many effective techniques, have been reported, such as including electromagnetic band gaps (EBGs) [1], modifying the radiating patches [2], re-constructing the ground planes [3], and resorting to a trident-shaped strip together with a tapered impedance transformer on the feedline [4].

As an alternative, in this talk, by integrating an asymmetrical single-wing and a symmetrical dual-wing filters into the feed-line section of a modified arc-slot UWB antenna, respectively, the broadside gain of the antenna in the upper portion of the UWB band is increased, because the magnitude of surface currents on the antenna radiation patch are improved. For example, the simulated broadside gains at 10 $\mathrm{GHz}$ are increased from $-3.89 \mathrm{dBi}$ to $4.16 \mathrm{dBi}$ for the single-wing antenna, and to $2.36 \mathrm{dBi}$ for dualwing antenna. Accordingly, the specific operation scheme of the filter-integrated antennas will be introduced and the differences between the asymmetrical and symmetrical versions will be illustrated in detail. Besides, the integration of filter into the antenna enhances the sharp cutoff performance at the two edges of the UWB frequency range. Moreover, the proposed co-design technology save the total size of the filter and antenna as a whole system effectively [3]. Finally, the experimental results, which are in agreement with the simulations, will be given in the talk to verify the proposed method.

\section{References:}

1. F. Fereidoony, S. Chamaani, and S. A. Mirtaheri, "UWB monopole antenna with stable radiation pattern and low transient distortion," IEEE Antennas Wireless Propag. Lett., vol.10, pp. 302-305, 2011.

2. R. Zaker, and A. Abdipour, "A very compact Ultrawideband printed Omnidirectional monopole antenna," IEEE Antennas Wireless Propag. Lett., vol. 9, pp. 471-473, 2010.

3. S.-W. Qu, J.-L. Li, J.-X. Chen, and Q. Xue, "Ultrawideband strip-loaded circular slot antenna with improved radiation patterns," IEEE Trans. Antennas Propag., vol. 55, pp. 3348-3353, Nov. 2007.

4. Q. Wu, R. Jin, J. Geng, and M. Ding, "Printed Omni-directional UWB monopole antenna with very compact size,” IEEE Trans. Antennas Propag., vol. 56, pp. 896-899, 2008. 